\title{
Monolobar Caroli'S Disease with Intrahepatic Cholangiocarcinoma-Diagnostic Dilemma in A Cystic Lesion of Liver
}

\author{
Senthilkumaran $\mathrm{GR}^{1}$,Prabhaharan $\mathrm{R}^{2}$,Benet Duraisamy ${ }^{3}$,Amudhan $\mathrm{A}^{4}$, Kannan \\ $\mathrm{D}^{5}$, Rajendran $\mathrm{S}^{6}$,NaganathBabu $\mathrm{OL}^{7}$ \\ Institute Of Surgical Gastroenterology, Rajivgandhi Govt. General Hospital, Madras Medical College, \\ The Tamilnadu Dr. MGR Medical University, Chennai, India.
}

Abstract: Caroli's disease is a rare biliary disorder characterised by multiple segmental dilatation of the intrahepatic bile ducts. Extent of liver involvement varies with monolobar variety occurring in $20 \%$ of cases. It can be associated with variety of complications of which cholangiocarcinoma remains a serious problem and can pose difficulty in diagnosis. We report a case of monolobar caroli's disease in a 67yrs old male diagnosed based on the imaging studies. During surgery, the affected lobe was found to harbor coexistent cholangiocarcinoma. Hepatic resection was offered as the treatment to manage both the conditions.

Keywords: Caroli`s disease, Caroli`s syndrome, Choledochal cysts, Cholangiocarcinoma.

\section{Introduction}

Cystic lesions of liver encompass a wider spectrum which range from very common to much rarer conditions and can also be either a benign or a malignant lesion. Though a distinction between most of the lesions can be made by clinical and radiological means, in few, the diagnosis can be arrived at, only through tissue diagnosis. We present an interesting case report of monolobar Caroli`s disease (CD) which during surgical resection was found to harbor cholangiocarcinoma (CCA).

\section{Case report}

A 67 years old gentleman presented with complaints of right upper quadrant pain of 1 week duration. $\mathrm{He}$ also had jaundice of same duration. He denied any history of fever, bowel disturbances, hematemesis or malena. General examination of the patient was normal except for mild icterus. Abdominal examination was non characteristic.

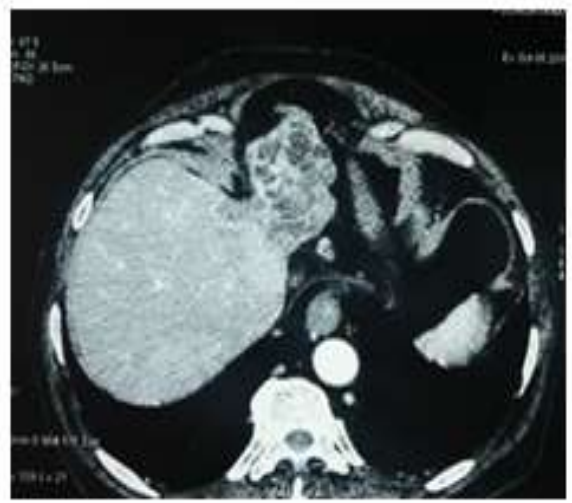

Fig. la: CT Scan Axial image showing cystic dilatation of biliary radicles of left lobe with lobar atrophy

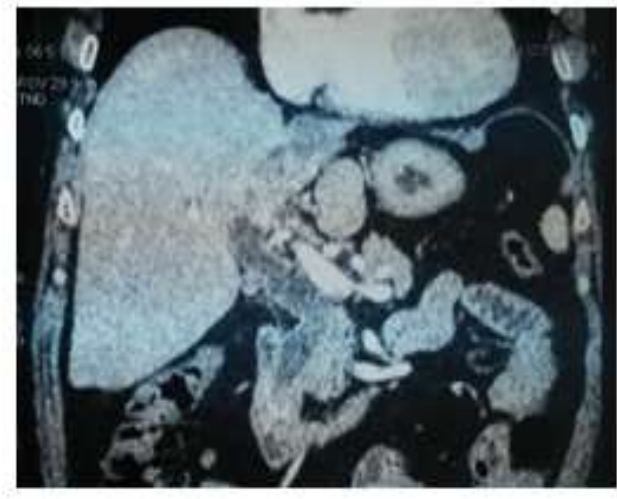

Fig.lb: CT Scan Coronal image showing fusiform dilatation of $\mathrm{CBD}$

Blood counts and renal parameters were normal; LFT was mildly deranged (Bilirubin Total-2.2mgs/dl; Direct-1.5mgs\%; SAP-121mgs \%) giving an obstructive picture. Ultrasound examination showed cystic lesions in left lobe of liver, gallbladder (GB) calculi with a dilated common bileduct(CBD) of $15 \mathrm{~mm}$ size. Upper GI endoscopy was normal. Portal doppler indicated a normal portal venous blood flow. Contrast enhanced CT scan and MRI scan of the abdomen were done which showed the following findings: Multi-loculated tubular/cystic 
dilatation of the intrahepatic biliary radicles of left lobe of Liver; atrophy of the left lobe of liver; right lobe of liver was normal; well distended gallbladder with few tiny calculi; fusiform dilatation of the CBD (16 mm) with a distal CBD calculi of size $8 \mathrm{~mm}$; portal vein and superior mesenteric vessels were normal; spleen normal in size; both kidneys were normal (Fig. 1\&2).

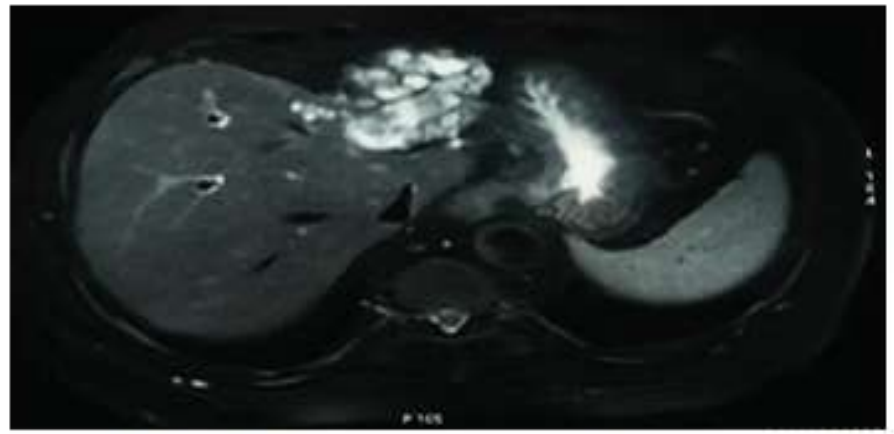

Fig.2: MRI scan showing T2 hyperintense dilated cystic biliary radicles

Diagnosis of Monolobar CD with CBD calculi was made. Since the disease process involved only the left lobe and the patient had lobar atrophy it was decided to proceed with resection. On laparotomy, left lobe of liver was found atrophic and there was diffuse fusiform dilatation of the CBD and CHD. On opening the left hepatic duct, a soft tissue fragment was noted, resembling tumor thrombi. This led to suspicion of hepatic malignancy. Left hepatectomy was done along with excision of the extrahepatic biliary tree since the CBD was dilated and appeared to be involved by disease process. Biliary reconstruction was done by right roux-en-y hepaticojejunostomy. Cut section of the hepatectomy specimen showed areas of cystic and saccular dilatation of the bile ducts along with grey white fleshy growth. Histology revealed moderately differentiated infiltrating cholangiocarcinoma of papillary type in the background of ectatic bileducts, consistent with $\operatorname{CD}($ Fig. 3 ). Resection margins were free of tumor. Patient recovered well in the postoperative period and was subsequently offered adjuvant chemotherapy consisting of 5-Flurouracil and Cisplatin.
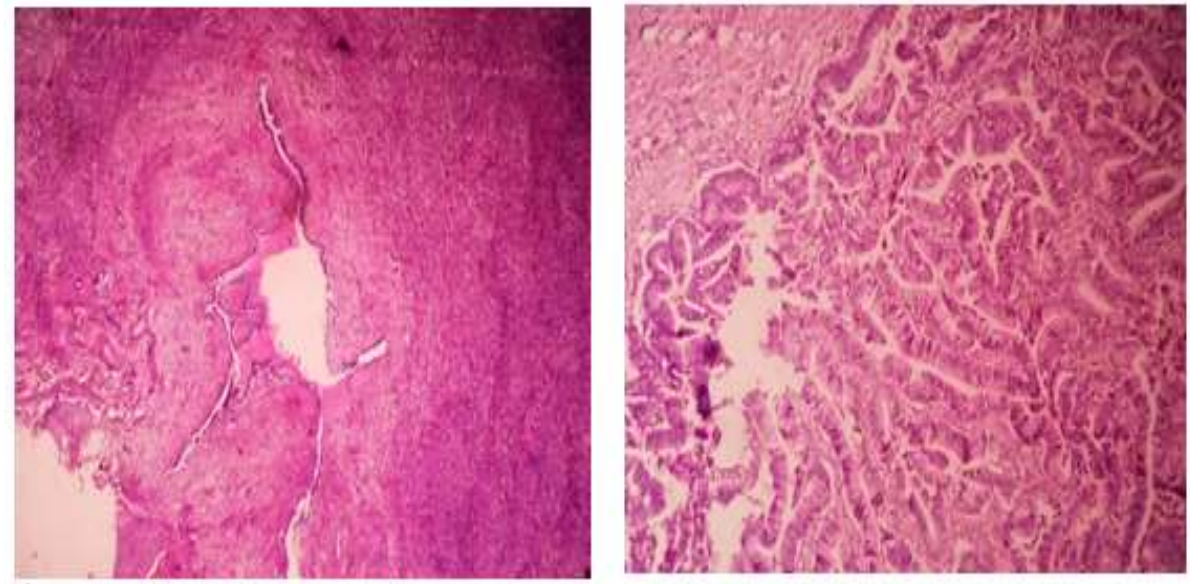

Fig. 3: Ectatic Bileduct with cholangiocarcinoma of papillary type (Low and High power view)

\section{Discussion}

Caroli`s disease is a rare congenital condition, characterised by multifocal, segmental, saccular or cystic dilatation of the intrahepatic bile ducts. It was first described by Jacques Caroli in 1958[1]. CD can either present as a diffuse disease involving both lobes of liver or rarely may be limited to one lobe in less than $20 \%$ of cases[2]. Monolobar CD commonly involves the left lobe of liver like it was in our case. Caroli`s syndrome (CS) indicates a complex variety where there is co-existing congenital hepatic fibrosis and/or polycystic kidney disease and is transmitted as an autosomal recessive disorder[3]. CD comes under the category of fibropolycystic diseases which also include polycystic liver disease, microhamartoma (Von meyenburgh complex), congenital hepatic fibrosis and choledochal cysts[4]. It has been categorized as Type V under the Todani et al classification of the choledochal cysts[5]. 
Clinical presentation is varied and includes right upper quadrant pain, jaundice, recurrent cholangitis, intrahepatic stones and abscesses. In CS, the clinical picture is dictated by presence of hepatic fibrosis and portal hypertension[3]. Laboratory investigations are nonspecific and reveal cholestasis and later in the disease, features of liver failure. Imaging studies like ultrasonography(US), computed tomography(CT) and magnetic resonance cholangiopancreatography(MRCP) reveal saccular or fusiform dilatation of the intrahepatic bile ducts and also help to establish communication between the cysts and the bileduct which is essential for the diagnosis of $\mathrm{CD}[6]$. Enhancing fibro vascular bundles in or along the margin of dilated ducts (the central dot sign) were found in $36 \%$ of patients with cross-sectional imaging studies. Endoscopic retrograde cholangio pancreatography (ERCP) and Percutaneous transhepatic cholangiography (PTC) are invasive imaging methods and are rarely indicated to confirm the diagnosis of CD. On occasion, CD can be accompanied by fusiform dilatation of the extrahepatic bileducts when the differentiation from the other choledochal cysts becomes difficult. In the case presented, extrahepatic bile duct showed fusiform dilatation with the CBD measuring $16 \mathrm{~mm}$ along with a secondary CBD calculus. The presence of diffuse fusiform dilatation of the extrahepatic bileduct measuring $3 \mathrm{~cm}$ or less in diameter combined with the characteristic intrahepatic ductal findings help to differentiate between the CD and other types of choledochal cysts[7]. Liver biopsies are not mandatory for the diagnosis of CD but can help to assess the extent of hepatic fibrosis in CS.

CCA is a more serious complication of CD.Occurrence of CCA in CD is 100 times greater than that in the general population with a reported incidence of 7-14\% [8,9]. CCA is more commonly identified in men with age more than 50 yrs. Diagnosis of CCA in the background of CD is difficult[7]. Due to laminar growth of the tumor, mass lesion may not be visualised in the cross sectional imaging. The treatment of CD is multidisciplinary. Drainage procedures like ERCP and PTBD can be of use in patients presenting with cholangitis. Surgical management of $\mathrm{CD}$ depends on the extent of the disease process. In localized or monolobar $\mathrm{CD}$, segmental or lobar hepatic resection can be done, especially, when associated with lobar atrophy[9,10]. Resection not only alleviates the septic complications but also prevents development of CCA in the affected lobe. In diffuse CD or CS, Liver transplantation is indicated as a final resort[10].

\section{Conclusion}

$\mathrm{CD}$ though a rarer entity, should be considered as a differential diagnosis in patients presenting with cystic lesion of liver. CCA can arise in the background of CD where the diagnosis can be difficult but is associated with better prognosis when curative resection can be offered.

\section{References}

[1]. Caroli J, Soupault R, Kossakowski J, Plocker L and Paradowska, [Congenital polycystic dilation of the intrahepatic bile ducts: attempt at classification],Sem Hop,34,1958, 488-495/SP.

[2]. Giovanardi RO, Monolobar Caroli's disease in an adult - Case report, Hepatogastroenterology, 50,2003, 2185-2187.

[3]. Yonem O and Bayraktar Y, Clinical characteristics of Caroli`ssyndrome, World J Gastroenterol, 13(13), 2007, 1934-1937.

[4]. Veigel MC, Prescott-Focht J, Rodriguez MG, Zinati R, Shao L, Moore CA and Lowe LH, Fibropolycystic liver disease in children,PediatrRadiol, 39,2009, 317-327.

[5]. Todani $\mathrm{T}$ et al, Congenital bile duct cysts: Classification, operative procedures and review of thirty-seven cases including cancer arisingfrom choledochal cyst,Am J Surg, 134, 1977, 263-269.

[6]. Miller WJ et al, Imaging findings in Caroli`s disease,AJR Am J Roentgenol, 165, 1995, 333-337.

[7]. Levy AD, Rohrmann CA Jr, Murakata LA and Lonergan GJ, Caroli`s disease: radiologic spectrum with pathologic correlation,AJR Am J Roentgenol, 179, 2002, 1053-1057.

[8]. Dayton MT, Longmire, WP Jr andTompkins RK, Caroli`s Disease: a pre-malignant condition?, American Journal of Surgery, $145,1983,41-48$.

[9]. Bockhorn M, Malagó M, Lang H, Nadalin S, Paul A, Saner F, Frilling A and Broelsch CE, The Role of Surgery in Caroli`s Disease,J Am CollSurg, 202(6),2006 June, 928-932.

[10]. Kassahun WT, Kahn T, Wittekind C, Mössner J, Caca K, Hauss J and Lamesch P, Caroli's disease: liver resection and liver transplantation- Experience in 33 patients,Surgery, 138(5),2005 Nov, 888-898. 